\title{
DISEÑO DE UN TEMA DE LENGUA Y LITERATURA INGLESAS A TRAVÉS DE FLASH Y DREAMWEAVER DE MACROMEDIA
}

\author{
(DESIGN OF A TOPIC OF ENGLISH LANGUAGE AND LITERATURE THROUGH FLASH AND \\ DREAMWEAVER BY MACROMEDIA)
}

\author{
$\mathrm{M}^{\mathrm{a}}$. Elena Gómez Parra \\ Antonio R. Raigón Rodríguez \\ Universidad de Córdoba (España)
}

\section{RESUMEN}

Este artículo pretende hacer una revisión de la metodología que se utiliza en los sistemas de educación a distancia, al mismo tiempo que trata el tema de la introducción del profesor en el uso de los sistemas hipermedia y/o multimedia para el desarrollo de la praxis docente. Ello supone un gran reto hoy día, avalado por la $U E$ en la formulación específica del Plan de Convergencia Europea para el sistema universitario de este nuevo milenio. Partimos de la premisa de que la formación del docente en las TICs es una condición sine qua non para el éxito del nuevo sistema universitario (formado por tutorías presenciales, virtuales y material de apoyo para su estudio y reflexión). Apoyándonos en las premisas del Marco Común Europeo de Referencia para las Lenguas, apuntaremos el esquema base del desarrollo de una lección perteneciente a un temario de Lengua y literatura inglesas para alumnos universitarios.

Palabras clave: TICs, metodología docente, alumnado y profesorado universitario.

\begin{abstract}
This article aims to review the methodology used in distance education, at the same time that it deals with the introduction of the teacher in the use of the hypermedia and/or multimedia systems for the teaching practice. This means a challenge, backed by the $E U$ and the European Convergence for the University curriculum of this new century. Our starting point is that teacher training in ICTs is a 'must' for the success of the new Spanish University model (in which distance and presence tutorials, as well as supporting materials for self-study and reflection are to be found). Taking into account the recommendations of the Common European Framework of Reference for Languages, we will propose the layout of a lesson belonging to a syllabus of English Language and Literature for university students.
\end{abstract}

Keywords: ICTs, teaching methodology, university students and teachers. 
Como es bien conocido, la implantación del Espacio Europeo de Educación Superior y la consecuente adopción del sistema de créditos europeos (ECTS) por parte de la Universidad española obligarán a una nueva concepción del método de trabajo utilizado tradicionalmente por parte del docente y del alumnado. En este cambio del que hablamos entran a formar parte por derecho propio las TICs y los métodos de enseñanza a distancia.

Así pues, pasamos a describir, brevemente, las tres partes que, a nuestro juicio, se verán afectadas por esta renovación universitaria española.

- En primer lugar, el profesorado universitario tiene que familiarizarse con las TICs, requisito indispensable en este nuevo modelo de enseñanza para el desarrollo eficaz de su praxis docente. La $U E$ y la consecuente implantación del sistema de crédito europeo en la Universidad española (en el que se valora en términos numéricos el trabajo personal del alumno) exigen un dominio claro y eficaz de las TICs tanto por parte del docente como por parte del discente. Está claro que, hoy día, el alumnado tiene pocos problemas para esta adaptación, pero creemos que no es éste el mismo caso para el profesorado, debido a una casuística muy variada entre la que podríamos citar la falta de tiempo para estas cuestiones, o la dedicación a temas más relacionados con la investigación personal, entre otras. Es decir, creemos que la adaptación rápida del profesorado a este tipo de instrumentos que, por otra parte, la sociedad del siglo XXI ya demanda se ha convertido en una tarea fundamental.

- El alumnado al que va dirigido este nuevo modelo universitario conforma la segunda parte de nuestro análisis. Como decíamos anteriormente, creemos firmemente que el alumnado tendrá menos problemas reales para adaptarse a esta nueva metodología discente debido, fundamentalmente, a una cuestión generacional. Con el fin de establecer un análisis práctico de la cuestión, propondremos el diseño de una lección perteneciente a un temario de la asignatura Lengua y Literatura inglesas para alumnos de $2^{\circ}$ curso de Maestro (con especialidad en lengua extranjera-inglés). Este curso se integra en el Aula Virtual de la Universidad de Córdoba y los alumnos a los que va dirigido pueden ver sus clases presenciales implementadas a través de este material, sirviéndoles de apoyo para el auto-estudio y ampliación de conocimientos sobre el temario visto en el aula previamente. Dicha lección, como ya avanzábamos, estará conformada por dos ejes fundamentales, (lengua y literatura) y de cuyo análisis y estructura nos ocuparemos más adelante. 
- Por último, la innovación de metodologías y el diseño de cursos virtuales completa este panorama que intentamos dibujar, dándole forma y cohesión a los dos elementos anteriores. El campo de las aplicaciones multimedia es un ámbito de innovación en el que se puede investigar y crear en materiales, diseño de mapas conceptuales, estructuras de enlaces, etc. En el diseño de nuestro curso hemos intentado introducir una variedad amplia de este tipo de elementos, que ejemplificarán ampliamente nuestro objetivo. Hablaremos de ellos en los apartados correspondientes al análisis del diseño y programación de nuestro curso.

\section{JUSTIFICACIÓN Y CONTEXTUALIZACIÓN}

La contextualización política y social de este estudio se perfila como condición sine qua non para, posteriormente, señalar el campo de actuación en el que se inscribirá el producto final diseñado. Comenzaremos, pues, por establecer, brevemente, el contexto educativo en el que este curso se desarrolla.

\section{La educación superior en el siglo XXI}

López Téllez (2002, p. 10) afirma lo siguiente, en referencia al papel de la educación en el siglo XXI: "Los primeros pasos dados en el ya recién estrenado siglo XXI nos indican que los grandes males de la humanidad: guerra, hambre, pobreza, etc., y consecuencia de ellos, la enfermedad, la ignorancia y el atraso en todos los órdenes de la vida, siguen manifestándose en distintos puntos del planeta como las grandes lacras de la humanidad. Ante esta situación, la educación se observa como uno de los elementos clave para resolver estos graves problemas, sobre todo, en momentos en los que la sociedad se ha hecho mucho más compleja y en la que se suceden cambios de muy diversa índole".

En efecto, una de las principales características de la sociedad del nuevo milenio es que se encamina hacia una sociedad del conocimiento, en la que el valor mayor de un país o de un colectivo humano estará, no tanto en su riqueza en materias primas, en su industria, en su comercio o en su capacidad de gestionar servicios, sino en su nivel de conocimiento, concebido como el conjunto de saberes individuales de sus ciudadanos puestos al servicio de la comunidad.

El nuevo marco de exigencias depende de la globalización y de la aparición de nuevas relaciones sociales. El Libro Blanco sobre la Educación y la Formación 
de la Comisión Europea precisa cuáles han sido los tres grandes impactos de nuestro tiempo sobre el mundo de la educación: la emergencia de la sociedad de la información, que está transformando la naturaleza del trabajo y de la organización de la producción; el fenómeno de la globalización, que incide sobre las posibilidades de creación de empleo; y, finalmente, la revolución científico-técnica, que crea una nueva cultura y que plantea acuciantes cuestiones éticas y sociales. La acción de estos tres factores ha influido radicalmente en la demanda y la provisión de los servicios de la Educación Superior.

Según el Informe a la UNESCO de la Comisión Internacional sobre la Educación para el siglo $X X I^{1}$, la educación constituye un instrumento indispensable para que la humanidad pueda progresar hacia los ideales de paz, libertad y justicia social, ya que el acceso a la educación transmite al hombre unos valores que mejoran su calidad de vida y la del conjunto de la sociedad. De esta forma, el proceso educativo se presenta como una de las claves del desarrollo económico y de la reducción de las desigualdades sociales, tanto de los países desarrollados como de los países en vías de desarrollo.

Si la educación tiene un papel imprescindible en el desarrollo de la sociedad, es de rigor preguntarse qué misión le corresponde dentro de ella a la educación universitaria. En este sentido, es fundamental tener en cuenta que otra característica de la época en la que estamos viviendo es que, sin una formación e investigación satisfactorias de nivel superior, ningún país puede asegurar un grado de progreso compatible con las necesidades y expectativas de su sociedad. En palabras del exDirector General de la UNESCO, Federico Mayor Zaragoza:

"En los umbrales de un nuevo siglo y de un nuevo milenio, somos testigos del extraordinario desarrollo de la enseñanza superior y comprendemos cada vez mejor su importancia vital para el desarrollo económico y social. Pero la Educación Superior se encuentra en crisis prácticamente en todos los países del mundo. El número de alumnos aumenta, pero la financiación pública disminuye, y aumenta también la distancia ya enorme que separa a países desarrollados y países en desarrollo en materia de enseñanza superior e investigación". (Mayor Zaragoza, 1995, p. 2)

Junto a este papel esencial de la Educación Superior hay que tener en cuenta la enorme transformación y expansión que el sistema universitario español (en particular) está sufriendo en los últimos años. A pesar detodas estas transformaciones, es posible establecer tres características principales comunes a todos los sistemas de Educación Superior de todo el mundo: 
- Expansión cuantitativa: ésta puede observarse en la matrícula de estudiantes en Educación Superior en todo el mundo, aunque los índices de crecimiento indican diferencias sustanciales según las regiones o países. Esta tendencia general se debe a varios factores: el crecimiento demográfico; los progresos significativos en el acceso a la enseñanza primaria y secundaria, que han dado lugar a un aumento del número de jóvenes que aspira a ingresar en el nivel educativo superior; el crecimiento económico experimentado en muchos países; y la conciencia de que el desarrollo tiene correlación con las inversiones en Educación Superior.

- Diversificación de las estructuras institucionales así como reorganización en los programas y las formas de estudio: las autoridades nacionales y las propias instituciones han introducido o comenzado a introducir modificaciones en las estructuras institucionales y las formas de Educación Superior, así como en los métodos de enseñanza, capacitación y aprendizaje. Uno de los resultados directos ha sido la diversificación en prácticamente todas las regiones del mundo.

Las razones de estos cambios son tanto externas como internas. Entre los factores externos cabe destacar: el aumento de la demanda social de Educación Superior y la necesidad de dirigirla a una clientela mucho más diversificada; los drásticos recortes en el gasto público, que han obligado a las instituciones a concebir programas distintos y más rentables; y las cambiantes necesidades del mercado laboral, que han obligado a los centros superiores a impartir formación en nuevos campos profesionales, tecnológicos y administrativos, y en nuevos contextos, debidos a la globalización y la regionalización de las relaciones económicas y comerciales así como del reciclaje de postgraduados.

Entre los factores internos que han llevado a una mayor diversificación y reorganización de los planes de estudio y las instituciones es necesario destacar el inmenso progreso de la ciencia, que ha estimulado el desarrollo de las disciplinas académicas y su diversificación. Por otra parte, el espectacular desarrollo de los conocimientos ha llevado a un aumento considerable del número de programas propuestos por los centros de Educación Superior. Una de las características de ese desarrollo es la interdependencia entre las distintas disciplinas científicas, y la creciente concienciación de que es preciso fomentar los planteamientos y métodos interdisciplinarios y multidisciplinarios de enseñanza, capacitación e investigación.

- Restricciones financieras: en la mayor parte de los casos, el aumento del número de estudiantes no se ha acompañado de aumentos reales en los recursos, 
obligando así a muchas instituciones a recortar sus presupuestos, programas de estudios, modernización de infraestructuras, recursos bibliográficos, cooperación internacional, e incluso, su personal académico.

Además de esto, el problema más importante al que se enfrenta la Educación Superior hoy en día es su adaptación a los cambios que la sociedad le exige, tanto en relación a las enseñanzas que imparte como a la investigación que realiza.

Desde su inicio, las Universidades han procedido tanto a difundir conocimientos como a preparar para la vida profesional. Pero al final del siglo XX, la situación adquirió unos rasgos nuevos y particulares. Actualmente, la capacitación profesional ha de permitir una continua renovación de los conocimientos para favorecer los cambios científicos y sociales en curso. Por otro lado, el aprendizaje especializado requerido para la capacitación profesional ya no se adquiere únicamente en la Universidad o en otras instituciones de enseñanza superior, sino que ha de ser necesariamente complementado con la propia práctica profesional. Además, si hasta hace pocas décadas los centros de Educación Superior se centraban aún en la formación de una elite, ahora la mayor sofisticación de las tareas que se pretenden desarrollar en los centros de trabajo ha ampliado considerablemente la población que ha de disponer de una formación superior.

Hoy día se espera que las instituciones de Educación Superior den una formación distinta de la que impartían unos años atrás. Los nuevos objetivos que ha de tener este tipo de educación, según la Comisión Europea, han de ceñirse al desarrollo de la capacidad de empleo teniendo en cuenta, sobre todo, la adquisición de las necesarias competencias que faciliten la creatividad, flexibilidad, capacidad de adaptación y la habilidad para aprender a aprender y a resolver problemas a lo largo de toda la vida. Según estos objetivos de la UNESCO (1995), los tres principales desafíos o retos que se le plantean a la Educación Superior en el futuro son: la pertinencia, la calidad y la internacionalización. Veámoslos:

- Pertinencia: se considera primordialmente en función del cometido y papel de la Educación Superior con respecto a la sociedad, y también desde el punto de vista de lo que la sociedad espera de dicho nivel de enseñanza. Por tanto, la pertinencia abarca cuestiones como las funciones de la Educación Superior en relación a la enseñanza, la investigación y los servicios conexos, así como de sus vínculos con el mundo del trabajo en sentido amplio, con el Estado y la financiación pública, y sus interacciones y responsabilidades con respecto al sistema educativo en su conjunto. 
Entre todos los aspectos que engloba la pertinencia, nos gustaría destacar la relación entre la educación universitaria y el mundo del trabajo. Esta relación viene determinada por dos tendencias paralelas: por un lado, la Universidad se ha transformado en un sistema que acoge a masas de estudiantes, lo que trae consigo un importante incremento del segmento de población profesionalmente cualificada y preparada para hacer frente a las exigencias del desarrollo económico; por otro, los egresados han de aceptar la necesidad de tener empleos que se transforman, de actualizar sus conocimientos y de adquirir nuevos conocimientos especializados. El mundo laboral está experimentando una transformación radical, y gran parte de los conocimientos específicos que adquieren los estudiantes durante su formación inicial pierden rápidamente actualidad. Por lo tanto, es esencial mantener relaciones constantes e interactivas con el sector productivo, integrándolas en la misión de los centros educativos superiores. Para ello, la Universidad debe transmitir y desarrollar en el individuo las cualificaciones necesarias que le permitan desarrollar la capacidad de adaptación a los cambios tecnológicos, la autodisciplina, la capacidad de decisión o de llevar a cabo su trabajo en equipos multidisciplinares en los que se integran profesionales de diferentes ámbitos.

En este sentido, se puede afirmar que la sociedad del nuevo siglo evoluciona hacia un modelo de educación permanente para todos, que irá sustituyendo gradualmente al modelo vigente de aprendizaje selectivo y concentrado durante un periodo de tiempo limitado. Así pues, sólo un sistema de acceso a la Educación Superior y de organización de la misma, suficientemente diversificado y flexible, podrá permitir la adaptación a un mercado de trabajo que se está transformando rápidamente.

- $\quad$ Calidad: la demanda de una Educación Superior más pertinente debe ir a la par con una voluntad general de mejora de la calidad; ésta ha de convertirse en una preocupación fundamental. A este respecto es necesario recalcar que la satisfacción de las necesidades de la sociedad y las expectativas que suscita la educación universitaria dependen, en última instancia, de la calidad del personal docente, de los programas y de los estudiantes, pero dependen igualmente de las infraestructuras y del entorno de la institución.

- Internacionalización: la internacionalización cada vez mayor de la Educación Superior obedece, en primer lugar, al carácter universal del aprendizaje y la investigación, viéndose fortalecida por los actuales procesos de integración económica y política y por la creciente necesidad de entendimiento intercultural. El incremento permanente del número de estudiantes, profesores e investigadores que estudian, dan cursos, investigan, viven y se comunican en el contexto internacional lo que demuestra claramente esta situación general. 


\section{Hacia un Espacio Europeo de Educación Superior (EEES)}

La construcción del Espacio Europeo de Educación Superior (EEES) es un proceso que se inicia con la Declaración de la Sorbona $(1998)^{2}$ y que se consolida y amplía con la Declaración de Bolonia (1999) ${ }^{3}$, en la que los ministros europeos de educación instan a la convergencia de los distintos sistemas de titulaciones con el fin de mejorar la transparencia y la compatibilidad de los estudios, títulos y diplomas. La convergencia propiciada por este proceso se basa en tres grandes ejes: la asunción de un modelo de titulaciones basado en dos niveles principales (grado y postgrado); la adopción de un sistema de créditos común para fomentar la comparación de los estudios y promover la movilidad de los estudiantes y titulados; y la promoción de la movilidad académica en Europa, dentro de un esfuerzo por mejorar la calidad de las instituciones a través de procedimientos constatados de evaluación y acreditación nacionales y supranacionales.

En el Comunicado de Praga (2001) ${ }^{4}$ se introducen algunos puntos adicionales relacionados con la formación continua y con el desarrollo de sistemas de garantía de la calidad y de mecanismos de certificación y de acreditación. Finalmente, la Cumbre de Jefes de Estado celebrada en Barcelona en marzo de 2002 supuso un hito importante en el proceso de construcción del Espacio Europeo de Educación Superior. Entre las conclusiones de la Presidencia del Consejo Europeo figura expresamente la de crear las condiciones prácticas necesarias para garantizar la movilidad a todos los que participen en los ámbitos de la educación, la investigación y la innovación, así como reducir los obstáculos normativos y administrativos al reconocimiento profesional. Se aprobó un programa de trabajo que, entre otras acciones, solicita la introducción de instrumentos concebidos para garantizar la transparencia de los diplomas y cualificaciones (ECTS, suplementos a los diplomas y certificados, CV europeo) y una cooperación más estrecha en la materia de diplomas universitarios en el marco del proceso La Sorbona-Bolonia-Praga.

El Espacio Europeo de Enseñanza Superior constituye un reto muy positivo a nuestro parecer. La introducción del crédito europeo como unidad del haber académico valora el volumen global del trabajo realizado por el alumno en sus estudios, así como el tiempo que se dedica a la evaluación o las tutorías, no computándose sólo las horas de clase. El diseño de los planes de estudio y las programaciones docentes se llevarán a cabo teniendo como eje de referencia el propio aprendizaje de los alumnos. El Suplemento Europeo al Título ayudará al reconocimiento más fácil y transparente por parte de otras Universidades y organismos europeos de la formación adquirida. Los estudios tendrán mayor transparencia y comparación con 
beneficios para toda la sociedad. Finalmente, la estructura de las enseñanzas que se cursen y los niveles de los títulos que se reciban al finalizar los estudios serán más homogéneos con los correspondientes títulos y enseñanzas del resto de países de la Unión Europea, favoreciendo así la movilidad e integración de los titulados en el mercado laboral.

Es en este ámbito donde los materiales virtuales cobran todo su sentido. En un sistema como el que acabamos de describir, el adecuado manejo de las TICs y la creación de materiales virtuales son condición sine qua non la Educación Superior no es posible.

\section{La Universidad en España}

\section{Panorama actual de la Universidad española}

Queremos contextualizar este trabajo en el ámbito de su desarrollo final, como decíamos anteriormente. Es por ello que creemos fundamental un recorrido breve por el panorama actual dela Universidad en nuestro país, queen los últimos años ha sufrido transformaciones importantes a todos los niveles ${ }^{5}$. Entre dichas transformaciones merecen especial mención las siguientes: el acelerado incremento del número de estudiantes que cursan estudios de Educación Superior, alcanzando una de las tasas de escolarización universitaria más altas de los países europeos; la creación de nuevas Universidades y centros universitarios; el proceso de descentralización política y administrativa al haber asumido las Comunidades Autónomas sus competencias en el ámbito universitario y por el propio ejercicio por parte de las Universidades del derecho constitucional de la autonomía universitaria; la ampliación y diversificación de la oferta educativa, tanto en titulaciones oficiales como en titulaciones propias; la potenciación y valoración de la actividad investigadora llevada a cabo en centros universitarios; y el incremento de la movilidad internacional de profesores y estudiantes.

Por otro lado, la integración de España en la Unión Europea ha determinado que estas modificaciones se adapten a un sistema de tendencia global en el que la Universidad desarrolla un papel sustancial.

El objeto de este apartado es abordar las claves del modelo universitario español tanto a nivel nacional como europeo, quedando así patente el contexto final donde tendría su aplicación final la lección que diseñamos en este trabajo. 


\section{Marco Legislativo: las funciones de la Universidad después de la LOU}

El Pleno del Congreso de los Diputados, en su reunión del día 20 de diciembre de 2001, aprobó el Proyecto de Ley Orgánica de Universidades (LOU) ${ }^{6}$. Con la entrada en vigor de esta controvertida ley, tras un largo y polémico debate social e institucional, se deja atrás el marco legal que ha regulado el funcionamiento de nuestras Universidades en los últimos veinte años, la Ley de Reforma Universitaria (LRU) ${ }^{7}$. En la exposición de motivos, que precede a los artículos de la ley propiamente dichos, se detallan los cambios ocurridos en el sistema universitario y en la sociedad en los últimos veinticinco años. Según el texto de la ley, ésta se presenta como un impulso renovador con la finalidad de "dotar al sistema universitario de un marco normativo que estimule el dinamismo de la comunidad universitaria". Los ámbitos de actuación de la ley se encuentran expresados en la exposición de motivos de la forma siguiente: "[...] se diseña la moderna arquitectura normativa que reclama el sistema universitario español para mejorar su calidad docente, investigadora y de gestión; fomentar la movilidad de estudiantes y profesores; profundizar en la creación y transmisión del conocimiento como eje de la actividad académica; responder a los retos derivados tanto de la enseñanza superior no presencial a través de las nuevas tecnologías de la información y de la comunicación como de la formación a lo largo de la vida, e integrarse competitivamente junto a los mejores centros de enseñanza superior en el nuevo espacio universitario europeo que se está comenzando a configurar".

En cuanto a los propósitos con los que nace la ley, el texto especifica: "Esta ley nace con el propósito de impulsar la acción de la Administración General de Estado en la vertebración y cohesión del sistema universitario, de profundizar las competencias de la Comunidades Autónomas en materia de enseñanza superior, de incrementar el grado de autonomía de las Universidades, y de establecer los cauces necesarios para fortalecer las relaciones y vinculaciones recíprocas entre Universidad y sociedad".

La $L O U$, al igual que ya hiciera la $L R U$, especifica en su artículo primero cuáles son las funciones de la Universidad. Dice así: "la Universidad realiza el servicio público de la Educación Superior mediante la investigación, la docencia y el estudio." Son funciones de la Universidad al servicio de la sociedad:

- "La creación, desarrollo, transmisión y crítica de la ciencia, de la técnica y de la cultura. 
- La preparación para el ejercicio de actividades profesionales que exijan la aplicación de conocimientos y métodos científicos y para la creación artística.

- La difusión, la valorización y la transferencia del conocimiento al servicio de la cultura, de la calidad de vida, y del desarrollo económico.

- La difusión del conocimiento y la cultura a través de la extensión universitaria y la formación a lo largo de toda la vida”.

Merece la pena detenerse brevemente a repasar las funciones que recoge la $L O U$ en el segundo apartado de su primer artículo. Nos detendremos en el tercer epígrafe de dicho apartado, mediante el cual la ley obliga al profesorado a permanecer en contacto continuo con la sociedad a la que pertenecemos y, en consecuencia, formar al tipo de profesionales que esa sociedad demanda. Ello conlleva una continua puesta al día, e incluso una posición de vanguardia, sin la cual la Universidad pierde gran parte de su razón de ser. No es concebible, hoy en día, una Universidad que vaya por detrás de la sociedad a la que se debe.

No es fácil, por tanto, la tarea de los docentes, y, desde luego, ésta no está exenta de una responsabilidad que va más allá de la enseñanza de las materias. En relación a esto, estamos firmemente convencidos de que nuestra sociedad no juzgará nuestra labor únicamente en virtud de cuánto sabe el titulado sobre tal o cual materia, sino también en virtud de qué tipo de ciudadanos salen de nuestros centros.

Queda así definida, por estas disposiciones, la misión de la Universidad, que no difiere en esencia, y pasadas siete décadas, de la que expresara $\operatorname{Ortega}^{8}$ (1930), al que podemos reconocer la actualidad de sus afirmaciones, según las cuales, la Universidad cumple cuatro misiones fundamentales:

- La enseñanza de las profesiones intelectuales.

- La investigación científica.

- La preparación de futuros investigadores.

- La transmisión de la cultura.

Sólo el tiempo permitirá juzgar si la recién estrenada $L O U$ es capaz de dotar a la Universidad de los medios necesarios para mejorar su calidad docente, investigadora y de gestión y para desarrollar sus funciones al servicio de la sociedad arriba recogidas. En cualquier caso, nuestra misión como docentes y como investigadores es hacer todo lo que esté en nuestra mano para hacer que la Universidad cumpla la que es para nosotros su misión fundamental: la creación y transmisión de la cultura. 
Integración del Sistema Universitario Español en el Espacio Europeo de Educación Superior

La $L O U$, en su exposición de motivos, establece como una de las finalidades del diseño de la nueva arquitectura normativa del sistema universitario español la de "integrarse competitivamente junto a los mejores centros de enseñanza superior en el nuevo espacio universitario europeo que se está empezando a configurar".

La integración del sistema universitario español en el Espacio Europeo de Educación Superior requiere de propuestas concretas que desarrollen los distintos elementos conceptuales definidos en las declaraciones europeas (Sorbona, 1998; Bolonia, 1999; Praga, 2001; Barcelona, 2002; Berlín, 2003) y recogidos por la LOU (título XIII, "Espacio Europeo de Enseñanza Superior"). En especial, resultan decisivas las medidas que deban adoptarse sobre el sistema europeo de créditos (ECTS), la estructura de las titulaciones, el Suplemento Europeo al Título (SET) y la garantía de la calidad.

- La implantación del sistema de créditos europeos ${ }^{9}$ : El crédito es la unidad de referencia sobre la que se estructuran y organizan los currículos formativos en la mayor parte de los países. El sistema de créditos europeos, conocido como ECTS (European Credits Transfer System), nace y se desarrolla con los programas de movilidad de estudiantes para dar respuesta a la necesidad de un sistema de equivalencias y de reconocimiento de los estudios cursados en otros países. El crédito europeo queda definido como la unidad de valoración de la actividad académica en la que se integran las enseñanzas teóricas y prácticas, así como otras actividades académicas dirigidas en el volumen de trabajo que el estudiante debe realizar para alcanzar los objetivos educativos. Su introducción en el sistema universitario español implica diferencias con respecto al crédito vigente, ya que el crédito europeo no es una medida de duración temporal de las clases impartidas por el profesor, sino una unidad de valoración del volumen de trabajo total del alumno, expresado en horas, que incluye tanto las clases (teóricas o prácticas), como el esfuerzo dedicado al estudio y a la preparación y realización de exámenes, así como a las tutorías. Es en este sistema donde nuestro trabajo cobra todo su sentido. Permitir al alumno la posibilidad de realizar un trabajo autónomo y a distancia es uno de los requisitos clave de los ECTS con la consecuente contabilización de dicho trabajo dentro de los créditos finales de la asignatura. La adopción de metodologías como la que aquí exponemos es el camino al que el Espacio Europeo de Educación Superior nos conduce. 
- $\quad$ Adaptación de la estructura de las titulaciones: El Gobierno establece, reforma o adapta las modalidades cíclicas de las enseñanzas universitarias con el esquema propugnado por la Declaración de Bolonia: Un primer nivel de grado que dará lugar a la obtención de un título con cualificación profesional en el mercado laboral europeo, y un segundo nivel de postgrado, para cuyo acceso será necesario haber superado el primero, y que podrá dar lugar a la obtención del Título de Máster y/o Doctorado.

- $\quad$ El primer nivel: El Grado: Los objetivos formativos de las enseñanzas oficiales de nivel de grado tendrán, con carácter general, una orientación profesional, es decir, deberán proporcionar una formación universitaria en la que se integren armónicamente las competencias genéricas básicas, las competencias transversales relacionadas con la formación integral de las personas y las competencias más específicas que posibiliten una orientación profesional que permita a los titulados una integración en el mercado de trabajo.

- El segundo nivel: El Postgrado: De acuerdo con lo establecido en la Declaración de Bolonia, el segundo nivel de las enseñanzas universitarias, para cuyo acceso se requerirá haber superado el primero, conducirá a la obtención de los títulos de Máster y/o Doctor.

- El Título Oficial de Máster: Los objetivos formativos serán más específicos que los de Grado y deberán estar orientados hacia una mayor profundización intelectual, posibilitando un desarrollo académico disciplinar e interdisciplinar, de especialización científica, de orientación a la investigación o de formación profesional avanzada.

- El Título de Doctor: Entre los objetivos fundamentales de la reforma propuesta se encuentra la revalorización de los estudios de doctorado y la mejora de los niveles de excelencia en el grado superior académico. El futuro del sistema universitario español pasa, en gran medida, por un incremento de los niveles de competitividad de estos estudios, así como por un mayor reconocimiento de la formación que proporcionan por parte de empresas e instituciones no académicas.

Esta etapa de formación consistirá en la elaboración y defensa de una tesis doctoral que deberá contener resultados originales de investigación. 
- El Suplemento Europeo al Título: El Suplemento Europeo al Título es una iniciativa europea auspiciada por el Consejo de Europa, la UNESCO y la Asociación Europea de Universidades. Constituye un elemento de transparencia cuyo objetivo fundamental es hacer comprensibles y comparables los títulos universitarios en Europa por medio de una información académica y profesional relevante para la sociedad, la Universidad y los empleadores.

El Suplemento Europeo al Título es un modelo de información unificado, personalizado para el titulado universitario, sobre los estudios cursados, su contexto nacional, y las competencias y capacidades profesionales adquiridas. Pretende ser un documento fácilmente comprensible, abierto a incorporar el aprendizaje a lo largo de la vida, acreditando los conocimientos adquiridos por cada persona en diferentes instituciones europeas de Educación Superior.

- Acreditación Académica y Calidad: La mejora de la calidad del sistema universitario es una pieza clave sobre la que pivota la construcción del Espacio Europeo de Educación Superior. La consecución del objetivo de lograr que Europa se convierta en lugar de referencia en el ámbito de la formación universitaria para estudiantes de todos los países pasa, necesariamente, por una garantía de calidad fundamentada en mecanismos y procesos de evaluación, certificación y acreditación.

La $L O U$ reconoce como uno de sus objetivos básicos la mejora de la calidad del sistema universitario en su conjunto, y en todas y cada una de sus vertientes. A tal fin crea la Agencia Nacional de Evaluación de la Calidad y Acreditación (ANECA) que, junto con los órganos de evaluación que puedan crearse en las Comunidades Autónomas, serán las responsables, en sus respectivos ámbitos, de llevar a cabo las políticas previstas de evaluación, certificación y acreditación, si bien la ANECA será la que intervenga en la elaboración de informes conducentes a la homologación de títulos de carácter oficial y validez en todo el territorio nacional. Así, la ANECA evaluará tanto las enseñanzas como la actividad investigadora, docente y de gestión, así como los servicios y programas de las Universidades.

Los sistemas de garantía de la calidad jugarán un papel importante para conseguir unos elevados niveles de excelencia que facilitarán la comparación y el reconocimiento de las calificaciones y títulos en toda Europa. Pero para ello será también necesaria una convergencia en los sistemas de garantía de calidad de los distintos Estados. 
La definición de criterios y estándares mínimos compartidos por las agencias responsables de asegurar la calidad y algunos criterios comunes sobre sistemas de acreditación y evaluación de estudios e instituciones serán elementos necesarios en la construcción del Espacio Europeo de Educación Superior. A tal fin, deberá desarrollarse al máximo la colaboración entre la ANECA y las agencias de otros países de la Unión Europea en el marco de la acreditación de títulos oficiales en los respectivos países.

Todo este complejo entramado aquí descrito nos sitúa y contextualiza en su justa medida el sistema de enseñanza que, ya hoy día, muchas instituciones de Educación Superior están aplicando en su praxis docente. No es concebible la Universidad del siglo XXI sin la aplicación práctica de las TICs en sus aulas. Permítasenos insistir: el docente universitario del siglo XXI tiene que estar al día, obligatoriamente, en lo que a avances tecnológicos se refiere. La lección de lengua y literatura inglesas que aquí se diseña no es más que uno de los muchos ejemplos que pueden ofrecerse con respecto a la nueva concepción de metodologías docentes y de aprendizaje.

\section{ESTADO DE LA CUESTIÓN Y ESTRUCTURA GENERAL}

El estado de la cuestión en este tema es muy complejo, porque puede ser abordado desde varios puntos de vista.

En primer lugar, el contexto universitario y la adopción del Espacio Europeo de Educación Superior por parte de la Universidad española nos ha permitido ya situar perfectamente el marco educativo y las características que marcarán la educación en este siglo. Por tanto, no abundaremos más en esta cuestión.

En otro orden de cosas, la metodología de la enseñanza de la lengua inglesa a distancia ofrece una amplia gama de materiales ya creados a disposición del alumno. Estos materiales son, en su mayor parte, muy innovadores y presentan un amplio abanico de posibilidades educativas.

Sin embargo, en lo concerniente a la enseñanza de la literatura inglesa a distancia, el campo está poco explorado y creemos que en este ámbito podemos ofrecer algún ejemplo de metodología interactiva y ejercicios multimedia sobre textos específicos que, entre otras cosas, animen al alumno a un acercamiento a la lectura de textos clásicos en literatura inglesa. 
Para aclarar los conceptos de los que hablamos, pasaremos a detallar la estructura general de la lección realizada y, al hilo de dicha explicación, iremos desgranando los avances metodológicos respecto a la enseñanza de la lengua y literatura inglesas a lo largo de este siglo.

La lección que hemos diseñado se basa en una estructura básicamente bipartita: lengua y literatura inglesas. Esta estructura nos permite dividir claramente los objetivos de aprendizaje de nuestros alumnos, cuyo contexto institucional previsto sería la Facultad de Ciencias de la Educación de la Universidad de Córdoba (España). El curso específico sería $2^{\circ}$ de Maestro, Lengua Extranjera (inglés).

Pasaremos a describir, uno a uno, los apartados diseñados y discutiremos brevemente el motivo que nos ha llevado a estructurar nuestra lección de esta manera.

\section{Enseñanza de la lengua inglesa}

En primer lugar y, como decíamos anteriormente, hemos optado por una estructura bipartita que nos permite separar claramente los objetivos de lengua y de literatura inglesas. De esta manera, el alumno tendrá claro desde el inicio qué quiere estudiar o practicar. Ello no significa, de ninguna manera, que el curso no permita la interacción de ambos apartados; todo lo contrario. Ambos ejes se encuentran perfectamente integrados y utilizaremos la información contenida en el apartado de lengua para el apartado de literatura y viceversa. Lógicamente, los contenidos lingüísticos son condición sine qua non para el entendimiento de los contenidos literarios y viceversa.

Nuestra lección de lengua inglesa supone un apoyo para el repaso de los tiempos verbales ingleses. Nos basamos en la premisa básica de que este tema ya ha sido visto en clase (sistema presencial) y utilizaremos el sistema virtual como soporte básico que permitirá al alumno repasar, complementar e indagar lo ya estudiado previamente en el aula.

Esta lección de tiempos verbales se compone de los siguientes apartados:

- Gramática (teoría sobre tiempos verbales y esquemas). Esta teoría no es más que un resumen de la ya vista en clase. No se trata, de ninguna de las maneras, del compendio general de todo lo que debe saber el alumno acerca de los tiempos verbales, sino sólo de un esquema general que sirva de recordatorio 
de la estructura general de los tiempos allí expuestos. Así, por ejemplo, no se introduce ex profeso el uso de estos verbos para que el alumno tenga la obligación de recurrir a las fuentes bibliográficas recomendadas y obligatorias del curso. Dentro del apartado teórico, se incide en la explicación y el análisis de dos tiempos verbales de especial dificultad: el pasado (en sus vertientes simple y perfecto) y el futuro (igualmente, en sus dos vertientes simple y perfecto).

- Ejercicios (de varios tipos y basados en la teoría anteriormente expuesta). Estos ejercicios inciden en la práctica de los conceptos anteriormente explicados y han sido diseñados según la metodología comunicativa de enseñanza de la lengua inglesa que, hoy por hoy, rige la praxis docente en nuestras aulas. El método comunicativo basa su metodología en las tareas, (tasks). Este enfoque basado en tareas para la instrucción de la lengua extranjera nace del interés del profesorado por las actividades comunicativas basadas en el significado y de la investigación realizada sobre los modelos de interacción de aula. Las tareas ('tasks') son actividades educativas que tienen su foco en el significado. Propuesta una actividad diversa, el éxito de una tarea se evalúa por la consecución de los objetivos propuestos, hecho que generalmente supone el uso efectivo de la lengua, como ocurre en la vida real. Así pues, la instrucción basada en tareas es considerada la concepción más fuerte del enfoque comunicativo en la enseñanza de la lengua (Communicative Language Teaching), constituyéndose ésta en la unidad articulatoria básica de diversos modelos de diseño de syllabus.

Este modelo de "consenso" sigue siendo polémico porque, aparentemente al menos, viene a establecer una confrontación terminológica entre corrección y fluidez (accuracy vs. fluency) o aprendizaje y adquisición (learning vs. acquisition), como si éstos, u otros similares, pudieran presentarse como fórmulas alternativas de aprendizaje de la lengua extranjera (L2) ${ }^{10}$. Evidentemente, el énfasis en cada uno de estos elementos binarios implica un modelo de enseñanza y articulación de las unidades docentes de tipo tradicional o de tipo procesual. En sus formas maximalistas, el primero de ellos exige un control por parte del profesor para conseguir la corrección como valor supremo de la enseñanza, mientras que el segundo modelo, persigue la fluidez como estrategia que el alumnado ha de desarrollar al objeto de conseguir su competencia. Hoy en día se asiste a una superación del movimiento pendular metodológico y se busca un enfoque ecléctico que, superando esta visión didáctica antagónica, establezca la creencia de que una auténtica fluidez tiene que estar fundamentada en una adecuada corrección formal. Es decir, el dominio de los conocimientos lingüísticos formales del sistema no puede ser un fin didáctico en sí mismo, sino un puente obligado para conseguir el conocimiento instrumental que facilite el uso comunicativo como función primordial de una lengua. 
Centrándonos ya en el modelo articulatorio de la 'tarea' como unidad de trabajo, su filosofía tiene como referente la teoría de los esquemas mentales (schemata) derivada del modelo cognitivo. Los esquemas no son necesariamente lingüísticos sino que pueden representar objetos, ideas, normas, acciones, sucesos, etc. Pero lo más importante desde el punto de vista de sus aplicaciones didácticas, reside en el hecho de que los esquemas no son exclusivamente de contenidos, sino también formales. Así pues, ambos tipos de conocimiento, formal e instrumental o de contenido, resultan muy útiles para el estudio de los procesos de adquisición de una L2 y cómo su enseñanza puede aplicarse a la construcción efectiva de la competencia comunicativa.

En este sentido, las tareas deben incluir ambos tipos de esquemas y ser diseñadas para que puedan ser analizadas en términos de bloques de conocimiento que han de ser dominados, activados, reciclados o reconstruidos significativamente por los alumnos. Como ejemplos de esquemas formales podrían citarse aquellos relacionados con el formato o codificación lingüística (léxico, estructura gramatical y discursiva) del tema seleccionado. Como ejemplo de esquemas instrumentales estarían los referidos a los procedimientos de codificación y descodificación del lenguaje mediante los cuales el alumnado lleva a cabo sus tareas, tales como: organizativos, de comprensión, de producción, etc.

Ningún grupo de esquemas involucrado en un bloque de conocimiento se construye de forma aislada sino como un sistema cognitivo interrelacionado o red. Y es esta red, que se crea o expande, la que permite enriquecer la trama de conocimientos lingüísticos y de contenido para posibilitar la negociación de nuevos significados utilizando la L2. Este proceso de construcción mental es uno de los fundamentos principales para considerar las tareas comunicativas como el contexto ideal o 'input total' - lingüístico y situacional - que contribuye, por una parte, al estímulo del interés de aprendizaje por parte del alumnado y, por otra, al desarrollo de tales redes que fomentan la construcción de la competencia comunicativa plena en el sentido de Canale y Swain.

En el proceso de llevar a cabo una tarea se activan de forma interrelacionada una serie de esquemas que actúan sincronizadamente: se incorporan nuevos elementos que se añaden a los ya existentes y se actualizan o construyen otros nuevos. Así pues, si se realiza un buen diseño de tareas comunicativas y capacitadoras, y su desarrollo tiene lugar en clase de forma adecuada, se producirá un proceso cognitivo continuado de integración y construcción de nuevos esquemas de conocimiento formal e instrumental, como un sistema eficiente de aprendizaje globalizado, coherente e interrelacionado. 
Las tareas comunicativas diseñadas tienen una amplia tipología que va desde ejercicios de gramática, completar huecos, hasta audiciones con varios ejercicios comunicativos en los que se pretende que el alumno comprenda el significado general del mensaje (lo que en terminología didáctica inglesa se reconoce con el término listening for gist) o complete una frase con un término específico que haya aparecido anteriormente en la cadena hablada (listening for comprehension).

- Juegos. Hemos querido complementar nuestras tareas con una serie de juegos que permitan un acercamiento algo más lúdico al aprendizaje de la L2. Estos juegos han sido diseñados siguiendo la misma metodología comunicativa de las tareas anteriormente expuestas.

- Humor. En este apartado hemos querido introducir elementos que forman parte de la cultura inglesa y que ayudan al alumno a comprender el entorno anglosajón. En él podemos encontrar frases, dichos, juegos de palabras y un poema humorístico. En los casos en los que la sátira o el humor reside en una palabra, se ha subrayado en amarillo para ayudar al alumno. En todos los casos, se añade un glosario específico que ayuda al alumno a comprender el humor inglés. Estos retazos humorísticos suponen un paso inicial en la conjunción de los apartados de lengua y literatura que hemos establecido en nuestro curso.

Entendemos que todos los apartados anteriores forman un conjunto bastante completo que permite al alumno un acercamiento integrador a la lengua y cultura inglesas. Es nuestro objetivo, como ya avanzábamos, ofrecer una metodología ecléctica que integre la lengua y la literatura desde un enfoque comunicativo. Si a todo ello añadimos la perspectiva que ofrecen las TICs en la enseñanza y el aprendizaje de la L2, obtendremos un panorama bastante esperanzador. No creemos que ésta sea la panacea para el aprendizaje de la L2, ningún enfoque ha demostrado serlo hasta la fecha, pero estamos convencidos de que la integración de estas disciplinas se mostrará eficaz en nuestro objetivo.

\section{Enseñanza de la literatura inglesa}

La literatura inglesa tiene menos tradición metodológica que la lengua inglesa. Dicho en otros términos, la lengua inglesa ha sido el objetivo de innumerables estudios y enfoques metodológicos, diseñados con el fin de facilitar y promover el aprendizaje de esta lengua. Sin embargo, la literatura inglesa no ha sido tan prolífica en este aspecto y, por tanto, la metodología de aprendizaje está aún por definir. 
No obstante, creemos que la integración de las TICs en el aprendizaje de la literatura inglesa aportará soluciones interesantes.

Nuestro curso, como ya decíamos, ofrece un apartado que se dedica al estudio de dos autores literarios. Al igual que ocurría anteriormente con el bloque diseñado para el estudio de la lengua, hemos querido ofrecer sólo una muestra de las posibilidades de actuación en este ámbito, y es por ello que se ofrece el estudio de dos autores de renombre universal en la literatura inglesa.

La elección de estos autores no ha sido hecha al azar. En nuestra elección han sido decisivos los siguientes factores:

- $\quad$ Autores de renombre (que inciten a la lectura y cuyo conocimiento sea amplio, de forma que la motivación intrínseca del alumno se dé de antemano).

- Autor masculino y autora femenina, que permitan un punto de vista genérico, capaz de abarcar y motivar al mayor número de lectores.

- Actual y no actual. Queremos que la literatura inglesa esté representada en una doble vertiente actual y tradicional.

El resultado de esta elección ha sido el estudio de los autores siguientes: Mary Shelley, autora del famoso Frankenstein perteneciente al siglo XIX y George Orwell, autor de 1984 o Animal Farm, perteneciente al siglo XX.

La estructura general de los dos apartados dedicados a la literatura ha sido el siguiente:

- Breve introducción biográfica del autor, que permitirá situar cronológicamente al alumno.

- Breve resumen de su obra más importante, de la que se extrae un fragmento.

- De dicho fragmento, se propone su lectura y análisis con algunos ejercicios que están diseñados para la comprensión textual y la aplicación gramatical de algunos puntos vistos en el apartado de nuestra lección dedicados al estudio de la lengua inglesa.

Esta estructura nos permite indagar un poco más en el estudio de la literatura inglesa ofreciendo un enfoque integrador entre el estudio lingüístico y el estudio literario, fundamental para nuestros alumnos que se convertirán en futuros maestros de inglés y que se verán favorecidos por dicho enfoque integrador. No queremos 
que nuestros futuros maestros sean meros transmisores del conocimiento, todo lo contrario; pretendemos que sean innovadores, integradores y buenos conocedores de la lengua, la literatura y la cultura inglesas. Todo ello está integrado por un hilo conductor que permite la conjunción de estos factores: las TICs como soporte del conocimiento y herramienta fundamental para la innovación y la transmisión de datos.

\section{LAS HERRAMIENTAS MULTIMEDIA}

Las herramientas que hemos utilizado y describimos a continuación han sido elegidas para desarrollar el curso de manera satisfactoria con la menor complejidad posible, pensando en la homogeneidad de criterios y lenguaje orientados a adaptación y ampliaciones sin problemas.

Con estos criterios, las herramientas empleadas han sido las propias de creación de hipertextos y sitios web, como MS FrontPage y Macromedia Dream Weaver, complementados con elementos de entrada que dinamizan los contenidos y facilitan sus enlaces como Macromedia Flash, con los que la animación, saltos y visualización se facilitan mediante la utilización de capas y escenas.

En el diseño se ha procurado la utilización de elementos (imágenes, archivos de sonido, animaciones y enlaces) de tamaño reducido, lo que dinamizaría la utilización del curso incluso a través de Internet.

El lenguaje utilizado (HTML y Visual Scripts) es el generado mediante las herramientas anteriores, que garantiza su interacción con otros sitios y cursos sin problemas de adaptación y legibilidad en cualquier otro sistema. Este método de trabajo pretende seguir la dirección tomada por los docentes que pretendan dinamizar su enseñanza y adaptarla a las necesidades del alumno respecto a recursos de información sin perder de vista en ningún momento el rigor temático de los contenidos.

\section{DISCUSIÓN Y CONCLUSIONES}

Este trabajo y la implementación del mismo con el diseño de una lección de lengua y literatura inglesas han pretendido ser el reflejo de una metodología integradora en la enseñanza y el aprendizaje de la lengua y la literatura inglesas. Como ya decíamos anteriormente, creemos que el Espacio Europeo de Educación Superior obligará a docentes y discentes a adoptar una nueva forma de enseñar y 
aprender la L2 respectivamente. Asimismo, el enfoque comunicativo en la enseñanza y el aprendizaje de la lengua ha demostrado, hasta la fecha, ser el más coherente y el más eficaz de cuantos han sido puestos en práctica.

Nuestro afán integrador en cuanto a lengua, literatura y cultura inglesas se refiere queda mostrado en la misma estructura del curso, en la que no se establecen dos apartados aislados, sino una perspectiva globalizadora.

Todo ello ha sido discutido y ejemplificado en anteriores apartados, por lo que creemos redundante insistir aquí.

Queremos, no obstante, resumir esquemáticamente las conclusiones finales a las que nuestro estudio nos ha conducido:

- Las TICs son una herramienta fundamental en la integración de los estudios universitarios en el Espacio Europeo de Educación Superior. Sin ellas, difícilmente entenderemos en toda su plenitud los nuevos créditos ECTS, la movilidad estudiantil o la evaluación permanente de la calidad en las titulaciones o el profesorado universitario. Es, pues, condición sine qua non para la adaptación completa de nuestros estudios.

- El estudio y la enseñanza de la L2 requiere por parte del docente de una metodología integradora, capaz de mostrar al alumnado universitario que el rigor científico, el dominio de la lengua y la capacidad de estudio no están reñidos con la imaginación, el humor y la calidad de los métodos. Es decir, en nuestra lección hemos intentado aunar factores tan diversos como el estudio de la lengua inglesa, su literatura y la cultura, a través de tareas tan diversas como los esquemas cognitivos, ejercicios prácticos, audiciones con aplicaciones prácticas, humor y juegos. Todo ello, esperamos, sea de utilidad a futuros profesores y maestros de inglés que entiendan que el nuevo milenio exige de la Universidad profesionales auténticos de la docencia, personal cualificado que quiera y pueda diseñar sus propios materiales de estudio, así como de desechar aquéllos que demuestren ser obsoletos o de poca utilidad en sus aulas.

- La Universidad y la Educación Superior del siglo XXI demandarán del docente la completa adaptación y actualización de conocimientos, así como de las necesidades reales del mercado de trabajo. En esta tarea, las TICs son una herramienta esencial que permitirá la continua puesta al día del docente en una sociedad que está en permanente cambio. 


\section{NOTAS}

1 Delors, J. et. al. (coord.) (1996). La educación encierra un tesoro. Informe a la UNESCO de la Comisión Internacional sobre la Educación para el siglo XXI.

2 http://www.eees.ua.es/documentos/declaracion sorbona.htm [consulta 2006, 14 de octubre].

3 http://universidades.universia.es/fuentes-info/documentos/bolonia.htm [consulta 2006, 14 de octubre]. http://www.ulpgc.es/index.php?pagina=espacioeuropeo\&ver=inicio [consulta 2006, 14 de octubre].

$5 \quad$ Informe Bricall. Universidad 2000.

6 Ley Orgánica 6/2001de 21 de diciembre de 2001 (B.O.E. de 24 de diciembre de 2001).

$7 \quad$ Ley Orgánica 11/1983 de 25 de agosto de 1983 (B.O.E. de 1 de septiembre de 1983).

8 Ortega y Gasset, J. (1930). "Misión de la Universidad", en Obras Completas (tomo IV). Madrid: Alianza Editorial.

9 Aprobado por RD 1125/2003 (BOE de 18 de septiembre de 2003).

10 De aquí en lo sucesivo utilizaremos el término L2 para referirnos a la lengua extranjera. El uso de este término abreviado es muy frecuente en los tratados de didáctica y metodología internacionales.

\section{REFERENCIAS BIBLIOGRÁFICAS}

Bowen, T.; Marks, J. (1997). Inside Teaching. Oxford: Heinemann.

Brown, R. (1991). Group work, task difference and second language acquisition. Applied Linguistics, 12, 1, 1-12.

Bueno Lajusticia, M. R. (1992). Un enfoque nuevo en la enseñanza del inglés. Revista Española de Lingüística Aplicada, 8, 167-175.

Burns, A. (1998). Collaborative Action Research for English Language Teachers. Oxford: O.U.P.

Bussé, L. (2003). Macromedia MX. Soluciones practices para Flash $M X$, Dreamweaver $M X$, Fireworks $M X$ and Coldfusion MX. Barcelona: Inforbooks.

Candlin, C. N.; Murphy, D. F. (eds.) (1994). Language learning tasks. Lancaster practical papers. English Language Education 7, London: Prentice-Hall.

Council of Europe (2001, $3^{\mathrm{a}}$ ed. 2002). Common European Framework of
Reference for Languages: Learning, teaching, assessment. Cambridge: C.U.P.

De la Serna Pozas, M. P. (1993). Diseño, desarrollo y análisis de proyectos académicos en L2, en: FernándezBarrrientos, M. (ed.), 82-91.

Ellis, R. (1985). Understanding Second Language Acquisition. Oxford: O.U.P.

Ellis, R. (1990). Instructed second language acquisition. Oxford: Basil Blackwell.

Estaire, S.; Zanon, J. (1990). El diseño de unidades didácticas en L2 mediante tareas: principios y desarrollo. Comunicación, Lenguaje y Educación 7-8, 55-90.

Johnson, K.; Johnson, H. (eds.) (1999). Encyclopedic Dictionary of Applied Linguistics. London: Blackwell.

Krashen, S. D. (1995). Issues in foreign language education. GRETA Revista para Profesores de Inglés, 3,2, 5-12. 
López Téllez, G. (2002). El profesorado de lenguas extranjeras ante los retos de formación del nuevo siglo. Ministerio de Educación, Cultura y Deporte. Lenguas extranjeras: hacia un nuevo marco de referencia en su aprendizaje. Madrid: Secretaría General Técnica, 9-27.

Mayor Zaragoza, F. (1995). Informe de Política para el Cambio y el Desarrollo en la Educación Superior. UNESCO.
Mc Farland, D. S. (2003). Dreamweaver MX. Madrid: Anaya Multimedia.

Peña de San Antonio, O. (2002). Dreamweaver MX. Madrid: Anaya Multimedia.

Widdowson, H. G. (1987). The roles of teacher and learner. ELT Journal 41,2, 83-88.

Windeatt, S. (2000). The Internet. Oxford: O.U.P.

\section{PERFIL ACADÉMICO Y PROFESIONAL DE LOS AUTORES}

$\mathbf{M}^{\mathbf{a}}$. Elena Gómez Parra. Licenciada en Filología Inglesa (Universidad de Granada, España). Doctora en Filología Inglesa (Universidad de Córdoba, España). Profesora del Dpto. de Filologías Inglesa y Alemana, adscrita a la Facultad de Ciencias de la Educación (Universidad de Córdoba, España). Vicedecana de Innovación, Recursos y Alumnado de la Facultad de Ciencias de la Educación (UCO-España). Coordinadora secciones inglés y alemán Ucoidiomas (Servicio de Idiomas de la UCO-España). Estancias de investigación recientes en la Universidad de Berkeley (California-USA) bajo la supervisión del Dr. Lakoff, la Universidad de Copenhague (Denmark), y la Canterbury Christ Church University (UK).

E-mail: ff1gopam@uco.es

Antonio R. Raigón Rodríguez. Licenciado en Filología Inglesa (University of Córdoba, España). Doctorando en Educación (Universidad de Córdoba, España). Profesor de Escuela Pública en Educación Primaria en Aurora (Illinois-USA) durante un año.

E-mail: antonio.raigon@uco.es

DIRECCIÓN DE LOS AUTORES:

Facultad de Educación

C/ San Alberto Magno, s/n

14071 Córdoba, España

Fecha de recepción del artículo: 17/10/06

Fecha de aceptación del artículo: 03/05/07 